\title{
Method of isolation of peptidoglycan as a basis for measuring murein-destroying activity of blood
}

\section{serum}

\begin{abstract}
Determining the activity of enzymes those destroy peptidoglican is the important part of the bacterial cell wall that can substantially help in diagnosis of bacterial infections. We propose the method of separation of a cell wall from gram-positive bacteria, which significantly simplifies and allows reducing cost of the isolation of peptidoglycan, which can be used as substrate for measuring activity of such peptidoglycan-destroying enzymes.
\end{abstract}

Keywords: gram-positive bacteria, peptidoglycan, enzyme activity, congo red

\author{
Volume I Issue 4 - 2014
}

\author{
Vikloryia Ziamko,' Vitaly Okulich² \\ 'Vitebsk State Medical University, Belarus \\ ${ }^{2}$ Department of Microbiology,Vitebsk State Medical University, \\ Belarus
}

Correspondence: Vikloryia Ziamko,Vitebsk State Medical University, Vitebsk, Belarus-210009, Belarus, Tel +375-29-146-0799, Email torinet@tut.by

Received: April II, 20I4 | Published: July 3I, 2014

\begin{abstract}
Abbreviations: AMP, antimicrobial peptides; PG, peptidoglycan; PLCR, peptidoglycan labeled with $2 \%$ solution of congo red; pkat, picokatals; $\mathrm{E}_{\mathrm{op}}$, optical density.

\section{Introduction}

Two classes of substances- endogenous antimicrobial peptides (AMP) and endogenous antimicrobial proteins play a very important role in the protection of organism against microbial agents. ${ }^{1,2}$ Antimicrobial proteins have rather large size containing more than 100 amino acids and most often show lytic enzymatic activity, ability to bind nutrients for bacterial cells or contain sites directed against specific microbial macromolecules. AMP have smaller size and as a rule, destroy the structure or the function of cellular membrane of microorganisms..$^{2-4}$
\end{abstract}

Nowadays, there are hundreds of AMP found in epithelial tissues, phagocytes and biological liquids. Some AMP are synthesized constantly, while the synthesis of others is induced in response to an infection or an inflammation. The majority of AMP is presented by cationic granules associated peptides with affinity to components of a microbial cellular wall, for example peptidoglycan. ${ }^{5}$

When monitoring a course of infectious and inflammatory disease in clinical laboratory practice, elevation of the AMP levels can be useful as marker of system activation of neutrophils. ${ }^{6}$ The knowledge of the role of enzymes, destroying peptidoglycan during infectious process is very important for understanding of deep mechanisms of interaction between microorganism and immune system of a macroorganism. This is necessary for development of new methods of diagnostics and treatment of infectious diseases.

In our opinion, there is scientific and practical interest in developing new methods of determination of serum activity of the peptidoglycan-destroying enzymes and assessment of their activity in course of various pathological processes.

Purpose: The purpose of this method is to isolate peptidoglycan from the cell wall of gram-positive bacteria and to analyze enzymatic activity of blood serum to destroy the isolated peptidoglycan in patients with purulent otitis.

\section{Methods}

\section{Isolation of peptidoglycan from gram-positive bacteria}

Isolation of $\mathrm{PG}$ from cell wall of gram-positive bacteria was carried out by a method proposed by V. Lvov, B. Pinegina and R. Khaitov in our modification. ${ }^{3}$ Microccocus luteus ATCC 10240 was used as a culture. The isolated PG, labeled with $2 \%$ solution of Congo red was used as a substrate for determining the activity of enzymes that destroy PG. ${ }^{7}$ The quality of the received PG has been determined by means of confocal microscope (Figure 1). We didn't remove teichoic acids, as it is known that their removal changes the specificity of bacteriolytic enzymes.

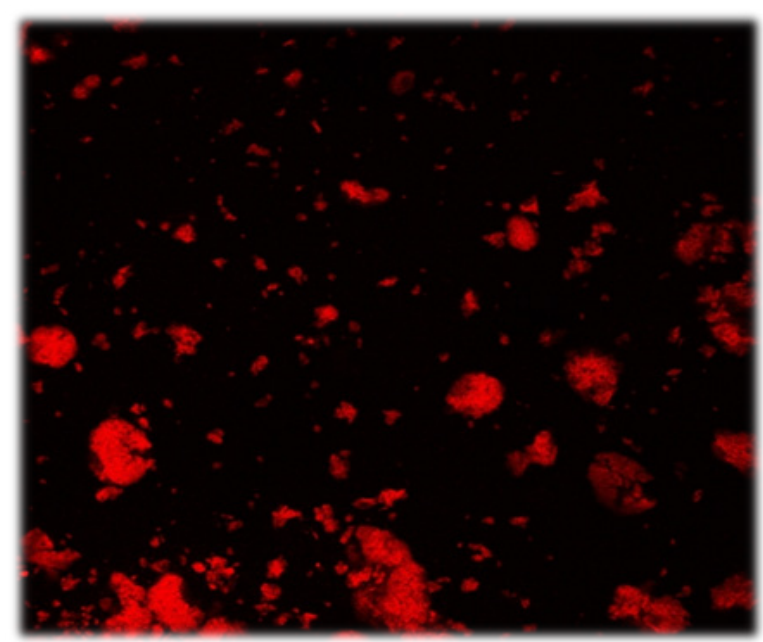

Figure I Substrate-Peptidoglycan labeled with Congo red. Diameter of particles from 2 to 9 microns (objective 40x, zoom=5).

Isolation of peptidoglycan is carried out as follows:

i. Bacteria were grown on meat-peptone agar. Overnight culture was washed twice with saline for removing components of the nutrient medium. It was centrifuged 1700 turns/min for $10 \mathrm{~min}$ and 2000 turns/min for $40 \mathrm{~min}$. 
ii. $20 \mathrm{ml}$ of $30 \%$ aqueous phenol pre-warmed at $90^{\circ} \mathrm{C}$ was added to the resulting suspension. The mixture was stirred for 20 minutes at a temperature of $65-68^{\circ} \mathrm{C}$ and cooled to $20 \pm 2^{\circ} \mathrm{C}$ and centrifuged 3 times for $10 \mathrm{~min}$ at 1700 turns/ min, removing the supernatant each time. At this stage, lipopolysaccharides (LPS), proteins, nucleic acids and other bacterial cell wall components non-covalently associated with PG, were removed. $30 \%$ aqueous phenol was used as an extractant at a temperature of $65-68^{\circ} \mathrm{C}$ (extraction by Westphal), since there is complete homogenization of a mixture of phenol and water at this temperature.

iii. Distilled water to a total volume of $300 \mathrm{ml}$ and $3 \mathrm{ml}$ of $100 \%$ acetic acid was added. The product obtained in the preceding step was stirred at $100^{\circ} \mathrm{C}$ for 3 hours to get free from trace amounts of LPS.

iv. After cooling, the precipitate was placed on a magnetic stirrer for 10 minutes and separated by centrifugation for 20 minutes at 2000 turns/ min. The precipitate was washed 3 times with water after each centrifugation.

v. Then dialysis was carried out at $21 \pm 2^{\circ} \mathrm{C}$, removing the dialysate 3 times (1 per a day) in a vessel and adding fresh buffer solution. Dialysis was performed to remove low molecular weight products that were formed during hydrolysis. $0.05 \mathrm{M}$ sodium acetate solution of $\mathrm{pH} 5.8$ was used as a buffer.

vi. The suspension was labeled with $0.5 \%$ solution of Congo red and it was washed twice with saline. The suspension was then centrifuged at 1000 turns/ min for 75 minutes after each wash to remove unbound solution of Congo red. ${ }^{6}$

Qualitative and quantitative characteristics of the isolated peptidoglycan were evaluated by confocal microscopy. Propidium iodide was added to the reaction mixture in the concentration of $20 \mathrm{~g} /$ ml. Further, a preparation of "hanging drop" was prepared. Stratified scanning was carried out immediately on confocal microscope Leica TCS SPE.

Scanning was performed in XYZ regimen by using 2 consequential spectral channels. These spectral channels were selected with characteristics such that there was no mutual overlap of the fluorescence from different dyes. The first channel (488nm laser, spectral detection area of 500-530nm) was used for excitation and detection of fluorescence FITC. The second channel (532nm laser, spectral detection area is $570-650 \mathrm{~nm}$ ) was used for propidium iodide. Scanning was performed with a resolution on the plane $150 \mathrm{~nm}$, with a distance of $500 \mathrm{~nm}$ between the layers. The time interval between scans was 3 minutes (Figure 1).

\section{Determining peptidoglycan-destroying activity of blood serum in patients with purulent-inflammatory diseases}

18 blood sera were taken from patients with purulent-inflammatory diseases and 18 sera were taken from healthy donors. Patients with purulent-inflammatory diseases were divided in 2 groups: 10 people with acute purulent otitis and 8 with chronic purulent otitis.

The serum was centrifuged 1.5 thousand/ min for 10 minutes. $300 \mu 1$ of PLCR solution and $100 \mu 1$ of serum were added into the first series of eppendorfs. $300 \mu 1$ of PLCR solution and $100 \mu 1$ of serum were pre-heated at $56^{\circ} \mathrm{C}$ for an hour in order to inactivate the complement. This solution was then added into the second series of eppendorfs. Samples containing Tris- $\mathrm{HCl}$ buffer at $\mathrm{pH} 7.4$ in an amount of $300 \mu 1$ and $100 \mu 1$ of serum served as a control. All the samples were incubated in thermostat at $\mathrm{t}=37^{\circ} \mathrm{C}$ for 24 hours. When enzymes in blood serum destroyed PG, Congo red became soluble changed its color from colorless to red with a maximum spectrum of absorption at a wavelength of $495 \mathrm{~nm}$. After incubation, the samples were removed from the thermostat and were centrifuged for $10 \mathrm{~min}$ (10thousand/ min; MICRO 120) for the remaining deposition of PLCR. 150 $\mu 1$ of the solution was taken from the supernatant in duplicate and transferred into the wells of 96-well polystyrene plate. The plate was placed in a multichannel spectrophotometer F300, where absorbance was determined in the wells in the wavelength of $492 \mathrm{~nm}$.

Interjacent result was expressed in units of optical density and was calculated as the difference between the optical densities of the test samples and their corresponding controls.

To convert the result into picokatals we used a formula obtained after constructing the calibration graph for the breeding of Congo red, in which the enzyme activity dependence from the optical density of the solution was reflected, assuming that after splitting of one substrate molecule, 1 molecule of Congo red goes into the solution (Figure 2).

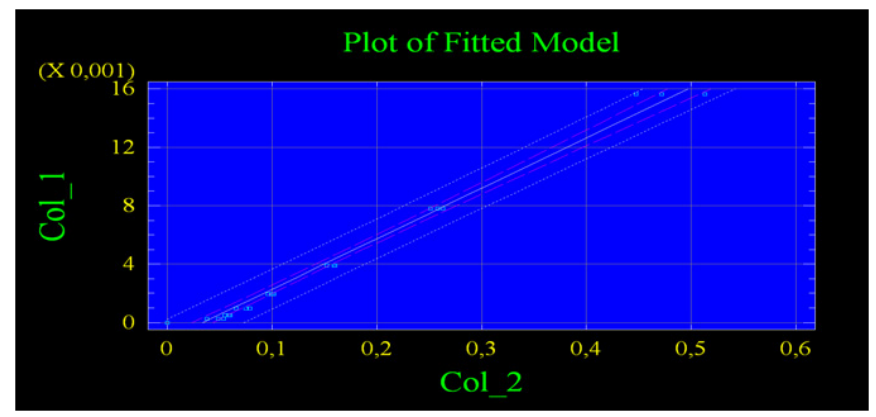

Figure 2 Graph of optical density of the solution concentration of Congo red. col_I, Concentration of Congo-red, $\mathrm{mg} / \mathrm{ml}$; col_2, Optical density of solution.

$Y=\left(-0.001+0.026 \times E_{\text {op }}\right) \times 9.921$

Where

Y: the desired result.

$\mathrm{E}_{\mathrm{op}}$ : optical density of the sample minus optical density of control.

Since the analysis of the data distribution showed their nonparametric distribution, statistic processing was performed by using the Kolmogorov-Smirnov test in which the differences were considered significant at $\mathrm{p}<0.05$.

\section{Results and discussion}

Experiment has revealed that peptidoglycan-destroying activity of blood serum in patients with purulent-inflammatory diseases was significantly higher in comparison with donors $(p<0.05)$. After that, the complement inactivation ability of enzymes to destroy PG significantly reduced $(\mathrm{p}<0.05)$. Results are presented in the Table 1 .

In our opinion, the results showed that the assessment of activity of peptidoglycan-destroying enzymes can be useful in diagnostics and treatment of infectious diseases. 
Table I Peptidoglycan- destroying activity of blood serum before and after complement inactivation in donors and patients with purulent otitis

\begin{tabular}{|c|c|c|c|c|c|}
\hline S. No & Group & $\mathbf{N}$ & Median, pkat & Percentile, pkat & Significance of differences \\
\hline I & Donors & 18 & 0.087 & $0.079-0.088$ & $P_{1-2}=0.03$ \\
\hline 2 & Patients with purulent otitis & 18 & 0.112 & $0.085-0.136$ & $P_{2-3}=0.02$ \\
\hline 3 & Patients with purulent otitis after complement activation & 18 & 0.076 & 0.047-0.099 & $P_{1-3}>0.05$ \\
\hline
\end{tabular}

\section{Conclusion}

i. The described technique allows to obtain the peptidoglycan component from the cell wall of Gram-positive bacteria and to determine antimicrobial activity of blood serum according to its ability to destroy peptidoglycan, which is one of the mechanisms of nonspecific resistance, allowing macroorganisms to fight against infection.

ii. It was found that the enzyme ability to destroy peptidoglycan was significantly higher in patients with purulent-inflammatory diseases $(0.112$ pkat $)$, than in donors $(0.087$ pkat, $\mathrm{p}<0.05)$.

iii. After complement inactivation, the enzyme ability to destroy peptidoglycan was significantly reduced $(0.076$ pkat, $\mathrm{p}<0.05)$.

\section{Acknowledgments}

We would like to thank Pleshkov FI, who contributed in the work and helped to translate it in English.

\section{Conflicts of interest}

Authors declare that there is no conflict of interest.

\section{References}

1. Kokryakov VN, Aleshina GM, Shamova OV, et al. Modern concept of antimicrobial peptides as molecular factors of the immunity. Med Acad J. 2010;10(4):149-160.
2. Budikhina AS, Pinegin BV. Defensins - multifunctional cations peptides of human. Immunopathology, Allergology, infectologist. 2008;2:S31S40.

3. Zemko V, Okulich V. Levels of enzyme activity of blood serum for the ability to destroy peptidoglycan in patients with purulent-inflammatory diseases. Regional European congress of biomedical laboratory science and the 4th Greek medical laboratory technologists conference: Athens; 2013.

4. Zemko V, Kiriluk O, Okulich V. Diagnostics of purulent-inflammatory diseases by studying of enzyme activity of blood serum for the ability to destroy peptidoglycan. Actual Problems of Biochemistry and Biotechnology. 2013;4:171-173.

5. Zemko V, Kiriluk O, Okulich V, et al. Method for isolating cell wall of Gram-negative bacteria: rats. Predlozhenie reg 31. 2013.

6. Zemko V, Kiriluk O, Okulich V. Act of introduction into the learning process 'Determing of enzyme activity of serum that destroy peptidoglycan" $1 ; 2013$.

7. Zemko V, Okulich V. Research activity by serum enzymes ability to break down peptidoglycan in patsietov with pyo-inflammatory diseases. Modern problems of infectious human pathology. Minsk. $2013 ; 6: 326-328$. 